\title{
Qubits Could Act as Sensitive Dark Matter Detectors
}

\section{A detector made from superconducting qubits could allow researchers to search for dark matter particles 1000 times faster than other techniques}

can.

\section{By Erika K. Carlson}

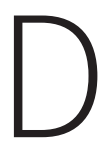
ark matter particles, such as axions and hidden photons, are theorized to occasionally "light up," converting into ordinary photons. This act potentially allows researchers to spot the otherwise invisible particles. Now, Akash Dixit of the University of Chicago and colleagues propose and demonstrate a way to make these detections with qubits [1]. Their calculations indicate that their technique is significantly more sensitive to detecting photons converted from hidden photons than conventional approaches are.

In the new method, a superconducting qubit watches for photons in a microwave cavity. The qubit can act as a detector because the energy required to excite the photon-something that researchers can monitor-depends on the number of photons in the cavity.

For any photon that appears, a conventional microwave-cavity detector would measure its position and momentum,

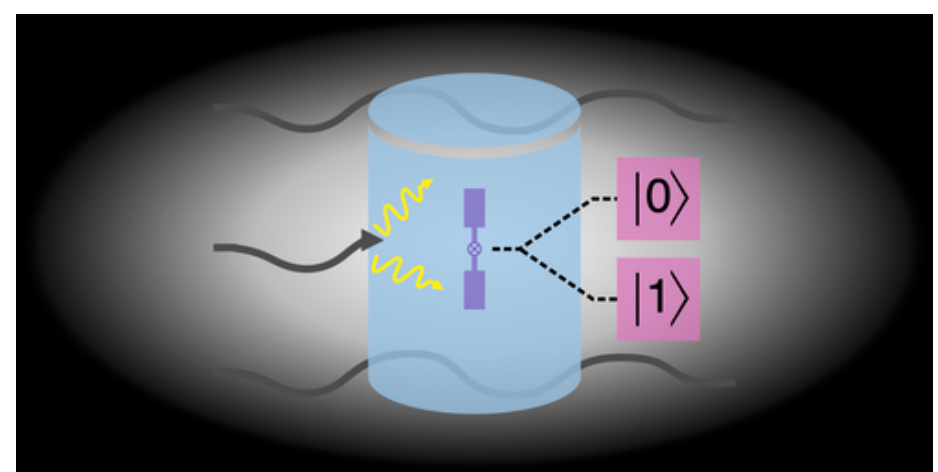

Credit: A. Dixit/University of Chicago introducing a significant level of noise because of the uncertainty principle. The qubit-based method of Dixit and colleagues needs to measure only one observable-the number of photons in the cavity-lessening the noise problem. Additionally, the qubit method leaves the photon intact, allowing for repeated measurements that reduce the likelihood of a false detection. These advantages could make dark matter searches roughly 1000 times faster than conventional microwave-cavity approaches.

Demonstrating the technique, Dixit and colleagues have already ruled out new regions of the parameter space for dark matter made of hidden photons. They say that with some modifications, the method could also be used to search for axions. For example, the device would need to work inside a magnetic field, which is necessary for converting incoming axions into photons.

Erika K. Carlson is a Corresponding Editor for Physics based in New York City.

\section{REFERENCES}

1. A. V. Dixit et al., "Searching for dark matter with a superconducting qubit," Phys. Rev. Lett. 126, 141302 (2021). 